\title{
Demokratisierung durch Partizipation? Die Mehrebenenbeteiligung an der Endlagersuche in Deutschland
}

\author{
Eva Krick
}

Online publiziert: 3. Dezember 2020

(C) Der/die Autor(en) 2020

Zusammenfassung Der Beitrag analysiert die Beteiligung an der Endlagersuche in Deutschland in Bezug auf ihre institutionellen Erfolgsbedingungen und demokratische Qualität. Im Zentrum steht die erste Phase der Endlagersuche, in der die „Endlagerkommission“ Input aus einer Vielzahl an Bürgerbeteiligungsforen erhielt. Die Studie zeigt, wie ausgeprägte institutionelle Komplexität, hohe normative Standards und passgenaue Koordinationsmechanismen dazu beitragen können, den zwei klassischen Schwächen partizipativer Verfahren - soziale Verzerrung und Resonanzlosigkeit - entgegenzuwirken. Aufbauend auf der inputorientierten Demokratietheorie und der empirischen Beteiligungs-, Deliberations- und Verhandlungsforschung wird insbesondere der Selektionsmodus der Zufallsauswahl hinterfragt und die bewusste Zielgruppenansprache als alternativer Weg zur Erreichung substanzieller Repräsentation diskutiert. Außerdem werden Aggregationsmechanismen und Rückkopplungskanäle typisiert, mit deren Hilfe eine Vielzahl an Stimmen integriert und in den politischen Prozess eingebettet werden kann.

Schlüsselwörter Bürgerbeteiligung · Demokratietheorie · Endlagerkommission · Deliberation · Minipublics

E. Krick (ه)

ARENA Centre for European Studies, University of Oslo, Oslo, Norwegen

E-Mail: eva.krick@arena.uio.no 


\title{
Democratisation by Means of Public Involvement? Multilevel Participation in the Search for a Nuclear Waste Repository in Germany
}

\begin{abstract}
This study analyses the public's involvement in the search for a nuclear waste repository in Germany in terms of the process's democratic quality and the institutional conditions of its success. It focuses on the initial phase of the search process, during which the "final storage committee" received input from various citizen participation forums. The study shows how institutional complexity, high normative standards, and well-designed coordination mechanisms can aid in moderating the classic weaknesses of participatory procedures-selectivity and lack of impact. Based on input-oriented democratic theory, participatory governance, deliberation, and negotiation studies in particular, the study questions the usefulness of random choice and discusses the targeted selection of affected groups as an alternative mode of participant selection. It also presents mechanisms of aggregation and transmission that can contribute to integrating societal voices and embedding them into the political process.
\end{abstract}

Keywords Citizen participation - Democratic theory $\cdot$ Final storage committee Deliberation · Minipublics

\section{Einleitung}

Mit der Erweiterung von Partizipationsmöglichkeiten sind in Deutschland hohe Erwartungen verbunden. In Zeiten zunehmender Elitenskepsis und abnehmenden Vertrauens in die repräsentativen Institutionen des politischen Systems werden insbesondere direktdemokratische Beteiligungsformate wie Referenden oder deliberative Foren in der öffentlichen Debatte als Quelle der „Demokratisierung“ gehandelt, die die demokratische Selbstbestimmung stärken.

Allerdings hat die Beteiligungsforschung wiederholt darauf hingewiesen, dass die Ausweitung von Partizipationsmöglichkeiten mit Schwierigkeiten verbunden ist: Gerade die oft als ,,innovativ“ oder ,,alternativ“ bezeichneten deliberativen Beteiligungsformate (wie etwa „Konsenskonferenzen“ oder „Bürgerdialoge“) sind in der Regel stark zugunsten privilegierter Bevölkerungsgruppen verzerrt und organisierte Gruppen sowie artikulationsfähige Individuen mit starkem Eigeninteresse dominieren (van Deth 2009; Fung 2006, S. 67; Irvin und Stansbury 2004, S. 59; Lijphart 1997, S. 1-2; Ritzi und Wagner 2016, S. 304-305; Schäfer und Schoen 2013, S. 100; Urbinati und Warren 2008, S. 405; Young 2000, S. 34). Hinzu kommt, dass deliberative Beteiligungsformate nicht selten wirkungslos bleiben. Oft gehen sie über die reine Informierung einer rezeptiv bleibenden Öffentlichkeit nicht hinaus, werden von den Beteiligten als „Pseudo-Partizipation“ (Pateman 1970) empfunden oder sogar als Manipulation abgelehnt (Irvin und Stansbury 2004, S. 59; Setälä 2017, S. 855). Politische Beteiligung, die berechtigterweise als „demokratisierend“ bezeichnet werden kann, ist also äußerst voraussetzungsvoll und hat besonders mit zwei Schwächen zu kämpfen: soziale Verzerrung und Resonanzlosigkeit. 
Vor dem Hintergrund dieser Herausforderungen untersucht die vorliegende Studie das bisher ehrgeizigste Beteiligungsformat auf Bundesebene - die Beteiligung an der Endlagersuche in Deutschland - in Bezug auf die demokratische Qualität des Prozesses. Gezeigt wird, wie institutionelle Komplexität und ausgeprägtes Engagement aller Seiten, aber auch passgenaue Koordinationsmechanismen dazu beitragen können, die beiden klassischen Schwächen partizipativer Verfahren auszugleichen.

Bei der Beteiligung an der Endlagersuche handelt es sich um einen hochkomplexen mehrstufigen Prozess, der diverse Beteiligungsarenen miteinander kombiniert und dem Ruf nach erweiterter Öffentlichkeitsbeteiligung weit entgegenkommt. Im Mittelpunkt der Analyse steht die erste Phase der Endlagersuche, in der die „Endlagerkommission“ Input aus unterschiedlichsten Bürgerbeteiligungskanälen erhielt. Diese umfassten zahlreiche deliberative, Face-to-Face-Bürgerkonferenzen, Worldcafés und Workshops, mit verschiedenen inhaltlichen Schwerpunkten und Zielgruppen (etwa junge Erwachsene, Beteiligungspraktiker, Fachöffentlichkeit, Regionenvertreter), mehrere Online-Diskussionsforen, Direktzuschriften per Mail und Brief sowie eine Dokumentenanalyse, die die Positionen der Anti-Atom-Bewegung einbezog. Nicht nur sind beachtliche Zeit- und Geldressourcen in diesen Prozess geflossen. Es wurden auch gleich mehrere Beteiligungsfirmen mit der Erarbeitung und Umsetzung des Beteiligungskonzepts beauftragt. Der Beteiligungsansatz wurde noch dazu laufend während des Prozesses evaluiert und angepasst. Er zielt auf ein Maß an Offenheit, Transparenz und Inklusivität, das auf bundesdeutscher Ebene und auch im internationalen Vergleich herausragt. Angesichts dieser hohen Ansprüche, aber auch im Wissen darum, wie voraussetzungsvoll gelungene Beteiligung ist, erscheint eine genaue Untersuchung der Beteiligungsqualität und ihrer Bedingungen im vorliegenden Fall lohnenswert. Schließlich ist es auch denkbar, dass die hohen Standards normative Dilemmata erzeugten oder die Mehrebenenstruktur Koordinationsprobleme nach sich gezogen hat.

Forschungsleitend stehen folgende Fragen im Vordergrund: Wie inklusiv und wie wirksam war der Beteiligungsprozess zur Endlagersuche? Welche institutionellen Mechanismen haben die Erreichung demokratischer Standards begünstigt und welche nicht - was waren Erfolgsbedingungen im vorliegenden Fall? Die normativen Maßstäbe zur Beurteilung des demokratischen Werts der Endlagersuche werden aus der beteiligungsorientierten Demokratietheorie abgeleitet und umfassen eine Gleichheits- und eine Wirkungsdimension. Die Erfolgsbedingungen werden induktiv gewonnen, wobei die dahinter liegenden institutionellen Mechanismen aber auch in Auseinandersetzung mit der Deliberations- und Repräsentationstheorie sowie empirischen Arbeiten zu Beteiligung, Konsultation und Politikberatung herausgearbeitet werden.

Zur Beteiligung an der Endlagersuche in Deutschland liegen mehrere sozialwissenschaftliche Beiträge vor, von denen sich einige auch schwerpunktmäßig mit der Endlagerkommission (Kalmbach 2016; Kamlage et al. 2019) bzw. der Bürgerbeteiligung an der Standortauswahl (Di Nucci 2016; Stolle 2006) beschäftigen. Die vorliegende Studie grenzt sich von diesen Arbeiten zum einen dadurch ab, dass sie explizit die Mehrebenenstrukur der Beteiligung - also die Bürgerbeteiligungsforen, die Endlagerkommission und die politische Entscheidungsebene - analysiert und ihr Zusammenspiel untersucht. Das bedeutet auch, dass der Wirkungsdimension 
von Beteiligung, der Frage der Resonanz, ein ebenso großer Stellenwert eingeräumt wird wie der Frage der Inklusivität, und das kann in der Beteiligungsforschung als eher ungewöhnlich gelten. Zum zweiten, und das ist entscheidend, kombiniert der Beitrag eine empirische Falluntersuchung mit einer normativen Analyse und einem erklärenden Ansatz. Das heißt der vorliegende Fall wird systematisch und kriteriengeleitet hinsichtlich der Beteiligungsqualität beurteilt und es werden institutionelle Bedingungen seines Erfolgs herausgearbeitet. Hauptsächlich zielt die vorliegende Untersuchung daher, sowohl im Bereich der Demokratietheorie als auch des Institutionendesigns, auf Theorieentwicklung ab: Die Einzelfallstudie ist also nicht in erster Linie aus Policy-Perspektive und als empirische Fallstudie interessant, sondern dient in hohem Maße der Konzipierung von Mechanismen, mit denen klassischen Schwächen erweiterter Beteiligung begegnet werden können. Sie leitet aus einem besonders ehrgeizigen und gelungenen Einzelfall institutionelle Erfolgsfaktoren ab, die auch für andere Beteiligungs- und Beratungsarenen in der Politikformulierungsphase handlungsleitend sein können.

Im Folgenden werden zunächst Grundkonzepte definiert und die normativen Merkmale ,guter“, demokratischer Beteiligung theoretisch skizziert (Abschn. 2). In einem zweiten Schritt, dem Hauptteil der Studie, werden diese Maßstäbe auf den Fall der Endlagersuche angelegt, und zwar zunächst auf die Endlagerkommission (Abschn. 3.1) und dann auf die zahlreichen Arenen der Bürgerbeteiligung, die Input für die Endlagerkommission geliefert haben (Abschn. 3.2.). In diesem Zuge werden auch die Mechanismen herausgearbeitet, die die Erreichung der Standards befördert bzw. behindert haben.

\section{Demokratietheoretische Maßstäbe guter Beteiligung}

Politische Beteiligung oder Partizipation steht gewöhnlich die Vielzahl an freiwilligen Handlungen (wie etwa der Wahlakt, die Parteimitgliedschaft, die Teilnahme an deliberativen Foren oder Demonstrationen), mit denen Betroffene, die nicht zum Regierungssystem im engeren Sinne gehören, auf politische Einflussnahme zielen (siehe etwa Brady et al. 1995, S. 271; Gabriel und Völkl 2005, S. 528). Die Konzepte der Bürger- oder Öffentlichkeitsbeteiligung legen den Fokus auf die breite und direkte, also nicht durch Stellvertreter vermittelte, Mitwirkung des ,normalen Bürgers“ oder der ,allgemeinen Öffentlichkeit“ an politischen Entscheidungsprozessen. Entsprechend werden hier Beteiligungsinstitutionen, die speziell auf die Einbeziehung der normalen Bürgerin oder des normalen Bürgers zielen, als Bürgerbeteiligungsforen, -arenen oder -formate markiert. Dies korrespondiert mit dem Konzept der Minipublics, d.h. ,deliberately constructed public spheres ‘ that ,convene citizens in selfconsciously organized public deliberations' (Fung 2003, S. 338-339). Als Beteiligungsarenen (auch: -formate, -verfahren, -foren) oder deliberative Foren werden hier allgemeine Institutionen der Politikberatung und Konsultation bezeichnet werden, in denen externe, zivilgesellschaftliche Akteure argumentationsbasiert interagieren und politische Empfehlungen entwickeln. Diese Konzepte beziehen sich also nicht nur auf Bürgerforen oder Minipublics, sondern umfassen auch sog. „Beiräte“ und „,Kom- 
missionen“, womit i.d.R. formalisiertere, stärker von professionellen Stakeholdern und Experten besetzte Gremien bezeichnet werden.

Das Konzept der demokratischen Beteiligung qualifiziert die politische Beteiligung in Bezug auf demokratische Schlüsselwerte wie Gleichheit, Selbstbestimmung oder Responsivität. „Participation is democratic when every individual potentially affected by a decision has an equal opportunity to affect the decision" (Warren 2002, S. 693; siehe auch Rowe und Frewer 2000, S. 12; Young 2000, S. 5-6). In diesem Zitat scheint eine Zweidimensionalität demokratischer Partizipation durch, die sich in eine Gleichheitsdimension und eine Einfluss- oder Wirkungskomponente gliedert, und die sich - implizit oder explizit - in der normativen wie empirischen Beteiligungsforschung und der sog. ,radikalen“ wie der gemäßigten Demokratietheorie findet (siehe etwa Marien et al. 2010, S. 140; Fung 2006, S. 66; Gabriel und Völkl 2005; Rowe und Frewer 2000, S. 12, 14; Pateman 1970, S. 42; Warren 2002, S. 693; Young 2000, S. 24).

Im Folgenden werden, zunächst für die Gleichheitsdimension und dann für die Wirkungsdimension, (a) die jeweiligen Maßstäbe demokratischer Beteiligung aus der Demokratietheorie hergeleitet und (b) operationalisiert. Das heißt es werden an den Kontext angepasste Indikatoren entwickelt, mit deren Hilfe die Qualität der Beteiligung in den untersuchten deliberativen Foren bestimmt werden kann. Darüber hinaus werden auch institutionelle Variablen genannt, die einen Einfluss auf die Erfüllung der Gütekriterien haben können (siehe auch Tab. 1). Wie diese im Einzelnen im vorliegenden Fall ausgeprägt waren und inwiefern sie der Erreichung demokratietheoretischer Maßstäbe förderlich waren, wird in der Fallanalyse herausgearbeitet und in den Schlussfolgerungen noch einmal fokussiert beleuchtet.

Tab. 1 Analyseschema zur Beurteilung der Beteiligungsgüte

\begin{tabular}{|c|c|c|}
\hline $\begin{array}{l}\text { Dimensionen } \\
\text { demokratischer } \\
\text { Beteiligung }\end{array}$ & (1) Gleichheitsdimension & (2) Wirkungsdimension \\
\hline $\begin{array}{l}\text { (a) Maßstäbe } \\
\text { demokratischer } \\
\text { Beteiligung }\end{array}$ & $\begin{array}{l}\text { Gleiche Beteiligung aller vom Thema } \\
\text { Betroffenen }\end{array}$ & $\begin{array}{l}\text { Resonanz des Beteiligungsverfahrens } \\
\text { im politischen Prozess }\end{array}$ \\
\hline \multirow{6}{*}{$\begin{array}{l}\text { (b) Gütekriterien } \\
\text { (Indikatoren) und } \\
\text { institutionelle } \\
\text { Voraussetzungen }\end{array}$} & Repräsentativität der Auswahl der & Beidseitige Beziehungen zwischen \\
\hline & Teilnehmenden & Beteiligungsarena und politischer \\
\hline & Institutionelle Voraussetzungen: & Ebene \\
\hline & $\begin{array}{l}\text { Zum Repräsentationsprinzip passen- } \\
\text { der Selektionsmechanismus }\end{array}$ & $\begin{array}{l}\text { Institutionelle Voraussetzungen: } \\
\text { Funktionierende Feedbackschleifen } \\
\text { und Rückkopplungskanäle }\end{array}$ \\
\hline & $\begin{array}{l}\text { Authentische (faire, inklusive, begrün- } \\
\text { dungsbasierte) Deliberation }\end{array}$ & $\begin{array}{l}\text { Durchsetzbare (konsensuale, konkrete, } \\
\text { policy-relevante) Vorschläge }\end{array}$ \\
\hline & $\begin{array}{l}\text { Institutionelle Voraussetzungen: } \\
\text { Illegitimität von Ausschlussmechanis- } \\
\text { men und kompetente, neutrale, faire } \\
\text { Moderation }\end{array}$ & $\begin{array}{l}\text { Institutionelle Voraussetzungen: } \\
\text { Funktionierende Aggregationsmecha- } \\
\text { nismen und Rückkopplungskanäle }\end{array}$ \\
\hline
\end{tabular}




\subsection{Die Gleichheitsdimension demokratischer Beteiligung}

(a) In Bezug auf die Gleichheit oder Inklusivität der Beteiligung soll hier der Maßstab gelten, dass die Perspektiven aller vom jeweiligen Problem Betroffenen im gleichen Maße in die Entscheidungsfindung einfließen (siehe etwa Fung 2013, S. 247; Rowe und Frewer 2000, S. 12-14; Warren 2002, S. 693; Young 2000, S. 5-6). ${ }^{1}$ Das Gleichheitskriterium gibt also nicht vor, dass die Beteiligung aller Betroffenen direkt, d. h. unvermittelt erfolgen muss. Ebenso denkbar ist die Beteiligung von Stellvertretern aller betroffenen Sichtweisen (siehe auch Irvin und Stansbury 2004, S. 60; Warren 2002, S. 686). Tatsächlich ist eine direkte Beteiligung aller Bürger*innen an allen sie betreffenden Entscheidungen bestenfalls in sehr kleinen Gemeinschaften vorstellbar. In Flächendemokratien (bzw. bei Fragen, die mehr als eine Handvoll Personen angehen) stößt sie dagegen leicht an Grenzen der Motivation und Organisierbarkeit (Fung 2006, S. 66; Ritzi und Schaal 2014, S. 245; Warren 2002, S. 686; Young 2000, S. 124). Dies gilt insbesondere für die Beteiligung in deliberativen Foren, die hier im Mittelpunkt stehen.

(b) Im vorliegenden Fall musste, wie noch zu zeigen ist, eine Teilnehmerauswahl getroffen werden, weil das bearbeitete Problem einen weiten Kreis an Bürger*innen betrifft und deliberativ, also argumentierend und im direkten Austausch, behandelt wurde. Während Wahlverfahren eine relativ große Zahl an Teilnehmenden integrieren können, bleibt sinnvolle Deliberation auf eine relativ kleine Gruppe von maximal etwa 30 Teilnehmern begrenzt (Krick 2013, S. 108; Ritzi und Schaal 2014, S. 256), weshalb man auch Minipublics und andere deliberative Foren als Repräsentationsinstitutionen verstehen muss (Ritzi und Schaal 2014, S. 259; s. auch Fung 2003; Krick 2014). Sobald aber eine Auswahl getroffen wird, stellen sich aus der Perspektive der Gleichheit oder Inklusivität Fragen nach der Repräsentativität, also danach, wie gut diese Auswahl die Grundgesamtheit abbildet (Fung 2006; Mansbridge et al. 2012; Urbinati und Warren 2008, S. 405).

Zwei Formen der Repräsentation sind für die vorliegende Untersuchung besonders wichtig (siehe Pitkin 1967; Ritzi und Schaal 2014; Urbinati und Warren 2008):

- Deskriptive oder statistische Formen der Repräsentation, bei denen die Frage im Zentrum steht, wie stark die Auswahl der Grundgesamtheit, d.h. dem jeweiligen demos, ähnelt.

- Substanzielle Formen der Repräsentation, die auf einer Verantwortlichkeitsbeziehung zwischen Repräsentant und Repräsentierten beruhen. In direkten Verantwortlichkeitsbeziehungen stehende Repräsentanten sind etwa Parlamentsabgeordnete oder Mitgliederorganisationen, die von ihrer „constituency“ autorisiert und zur Rechenschaft gezogen werden. ${ }^{2}$

\footnotetext{
1 Für die Beurteilung dieses Maßstabs ist wichtig zu bedenken, dass der Kreis der Betroffenen vom jeweils bearbeiteten Thema abhängt und zum Kreis der Teilnehmenden in Beziehung gesetzt werden muss.

2 Verantwortlichkeitsbeziehungen können auch auf indirektem Wege hergestellt und durch glaubwürdige Vertretung und öffentliche Anerkennung legitimiert werden, wie z. B. Saward (2010) ausführt (siehe auch Warren 2002).
} 
Während deskriptive Repräsentation am ehesten durch Zufallsauswahlen wie etwa Losverfahren oder Quotenstichproben hergestellt werden kann, ist die gezielte Auswahl (,targeted selection“) stärker, wenn es darum geht, eine Gruppe zusammenzustellen, deren Mitglieder als legitimierte Stakeholder der betroffenen Sichtweisen, Interessen und Positionen gelten können oder wenn es darum geht, Personen mit relevanter Expertise auszuwählen (siehe auch Fung 2003; Ryfe 2005; Warren 2002).

Neben der Repräsentativität kommt bei deliberativen Foren das Legitimationskriterium der fairen, inklusiven oder auch authentischen Deliberation hinzu (Dryzek 2001, S. 651; Ritzi und Schaal 2014). Dies betrifft die De-facto-Berücksichtigung aller Teilnehmenden an der kollektiven Entscheidung der Arena, die durch zahlreiche Ausschlussmechanismen eingeschränkt werden kann. Dazu zählen etwa Entscheidungsregeln, die einzelne Stimmen marginalisieren oder der Ausschluss bestimmter Themen von der Agenda (siehe auch Fung 2006; Krick 2013; Krick und Holst 2018; Mansbridge et al. 2012; Young 2000). Eine Schlüsselrolle bei der inklusiven Gestaltung der Interaktion kommt in deliberativen Foren dem Vorsitzenden oder Moderator zu (Krick 2013, S. 234-240).

\subsection{Die Wirkungsdimension demokratischer Beteiligung}

(a) In der Wirkungs- oder Effektivitätsdimension geht es darum, dass alle Betroffenen die Chance haben, Einfluss zu nehmen auf die jeweilige politische Entscheidung (Fung 2006, S. 66; Krick 2014, S. 11; Marien et al. 2010, S. 140; Rowe und Frewer 2000, S. 12, 14; Warren 2002, S. 693). Auch hier muss die Beteiligungspraxis keine direkte, unmittelbare Wirkung entfalten, damit das Kriterium erfüllt ist; wichtig ist vielmehr, dass es sich nicht um „Alibiveranstaltungen“ und „Pseudopartizipation“ (Pateman 1970, S. 68) handelt, sondern um ernstgemeinte Beteiligungsverfahren, die politische Resonanz entwickeln können (siehe auch Fung 2006, S. 66; Gabriel und Völkl 2005, S. 529; Krick 2014, S. 11; Marien et al. 2010, S. 140; Pateman 1970, S. 68-69; Rowe und Frewer 2000, S. 3, 12, 14; Warren 2002, S. 693; Young 2000, S. 24). Keineswegs ist ernstgemeinte, potenziell einflussreiche Beteiligung mit unmittelbarer Entscheidungsmacht gleichzusetzen. Gerade deliberative Foren wirken i.d.R. konsultativ, bekommen also keine Direktentscheidungsgewalt übertragen, können aber dennoch auf indirektem Wege oder zeitversetzt Einfluss auf Politikwandel und Agendasetting entwickeln. Ebenso wenig sind die wirkmächtigsten Beteiligungspraktiken normativ überlegen oder nur diese Formen als Partizipation zu bezeichnen. ${ }^{3}$ Tatsächlich sollte aus demokratietheoretischer Sicht keinem Beteiligungsformat im Vorhinein Einfluss garantiert werden, sondern dies davon abhängig gemacht werden, wie die Beteiligungspraxis jeweils in Bezug auf Normen der Input-Legitimation wie Inklusivität und Transparenz (Fung 2003, 2006; Lafont 2015), aber auch epistemische Qualitäten wie Problemlösungsfähigkeit oder PolicyRelevanz abschneidet (Krick 2018).

\footnotetext{
3 Diese Annahme liegt der einflussreichen „Partizipationsleiter“ zugrunde, die 1969 von Arnstein entwickelt wurde, mittlerweile aber sowohl in der Demokratietheorie (siehe Fung 2006, S. 66-67) als auch in der Beteiligungspraxis kritisch gesehen wird (siehe etwa Berlin Institut für Partizipation 2018; Deutsche Arbeitsgemeinschaft Endlagerforschung 2016).
} 
(b) Eine wichtige Voraussetzung für Resonanz sind beidseitige Beziehungen zwischen dem Beteiligungsformat und dem Empfänger des Inputs (Fung 2006). Die zentralen Stellschrauben, durch die diese Beziehung institutionalisiert wird und politische Resonanz ermöglicht werden kann, sind funktionierende Rückkopplungskanäle oder Feedbackschleifen. Es müssen Kanäle bestehen, über die Vorschläge aus der Beteiligungspraxis in den politischen Prozess einfließen, sowie Kanäle, über die der Umgang politischer Entscheidungsträger mit solchem Input transparent gemacht wird und von der Zivilgesellschaft überprüft werden kann (Brown 2006, S. 213; Lentsch und Weingart 2011, S. 371; Rowe und Frewe 2000, S. 15). Das Feedback politischer Entscheidungsträger darf dann auch in der Erklärung bestehen, dass mit den Vorschlägen aus der Beteiligungsarena wenig anzufangen ist, weil sie bspw. das Problem nicht ausreichend adressieren, uninformiert oder nicht durchsetzbar sind. Auch kann es sicherlich zur Resonanz der Vorschläge beitragen, wenn bereits während der Deliberation Vertreter der Exektuive beratend zur Seite stehen oder die Umsetzbarkeit der Vorschläge in der Legislativarena prüfen.

Die Durchschlagskraft von Beteiligungsforen wird außerdem durch Faktoren beeinflusst, die in den Vorschlägen selbst liegen. Wenn ein deliberatives Forum die Positionen aller Teilnehmenden zu einer gemeinsamen Stimme integriert, die im besten Fall von einem breiten Konsens getragen wird (Dryzek 2001, S. 651; Fung 2006, S. 68; Krick 2013), und es außerdem konkrete, policy-relevante und politisch umsetzbare Vorschläge vorlegt, dann erhöht das die Legitimität des Forums und die Einflusschancen (Haas 2004, S. 575; Krick 2013, S. 126; Lentsch und Weingart 2011, S. 367). Der Erreichung solch durchsetzbarer Vorschläge förderlich sind etwa funktionierende Aggregationsmechanismen (wie Abstimmungs- oder Konsensverfahren), eine kollektive Identität und Konsensorientierung der Gruppe sowie wiederum die Rückkopplung mit dem Regierungssystem während der Beratungen.

Die hier entwickelten Gütekriterien werden im Folgenden systematisch auf den empirischen Fall der Beteiligung an der Endlagersuche angewandt. Es wird außerdem analysiert, welche institutionellen Faktoren der Zielerreichung förderlich bzw. hinderlich waren. Ziel der Analyse ist zum einen Processtracing, also die Rekonstruktion und Durchdringung der Fallgeschichte, zum zweiten die Bewertung der Beteiligungsgüte im vorliegenden Fall und zum dritten die Herausarbeitung von Bedingungen gelungener Beteiligung. Damit werden also sowohl Theorieentwicklung als auch die Erklärung der Zusammenhänge im untersuchten Fall angestrebt. Die Fallstudie beruht auf der Auswertung von Dokumenten wie dem Kommissionsbericht, Sitzungsprotokollen, Dokumentationen der Beteiligungsformate, Evaluationsberichten und Bundestagsdrucksachen, ${ }^{4}$ auf Analysen von Videomitschnitten und teilnehmenden Beobachtungen bei Kommissionssitzungen sowie acht Experteninter-

\footnotetext{
4 Von besonderer Bedeutung für das Processtracing sowie die normative Analyse der Beteiligungsqualität waren folgende Dokumente: der Kommissionsbericht, die Kommissionsgeschäftsordnung, das Standortauswahlgesetz (StandAG), Beratungsunterlagen zu den Kommissionsitzungen, Auftragsgutachten und Sitzungsprotokolle, wenn diese den Beteiligungsansatz betrafen, Bundestagsgesetzentwürfe, -änderungsanträge und -plenarprotokolle zum StandAG, Dokumentationen der Beteiligungsformate, Beteiligungskonzept und -evaluation, Stellungnahmen der Zivilgesellschaft sowie Pressestimmen zum Thema Endlagerung, Kommission und Beteiligung im Besonderen.
} 
views mit Kommissionsmitgliedern und mit einigen, mit dem Prozessmanagement betrauten Verwaltungsbeamten und externen Dienstleistern.

\section{Fallstudie: Die Beteiligung an der Endlagersuche}

Die Suche eines Endlagers (oder Standorts) für hoch radioaktiven Abfall in Deutschland ist in zwei Phasen aufgeteilt: erstens: die Einigung auf ein Verfahren für die Endlagersuche und die Kriterien für die Endlagerauswahl und zweitens das darauffolgende Standortauswahlverfahren. In beiden Phasen legt der Gesetzgeber großen Wert auf die intensive gesellschaftliche Beteiligung, um ,eine Lösung [zu] finden, die in einem breiten gesellschaftlichen Konsens getragen ist und damit auch von den Betroffenen toleriert werden kann. Hierzu sind Bürger*innen als Mitgestalter des Verfahrens einzubeziehen“ ( $\$ 5$ Standortauswahlgesetz [StandAG 2013]; siehe auch StandAG $2013 \S 1$ Abs. 1; Bundestags-Plenarprotokoll 18/179, 182-185).

Hintergrund dieses Bekenntnisses zu Beteiligung und Konsens im StandAG sind die tiefen politischen Gräben und die jahrzehntealten, verhärteten Konflikte rund um den Standort Gorleben sowie das ausgeprägte gesellschaftliche Misstrauen gegenüber staatlichem Handeln im Bereich der Endlagersuche. ${ }^{5}$ Der Salzstock Gorleben wurde 1977 in Abstimmung zwischen Bundes- und Niedersächsischer Landesregierung als Atommüllendlager festgelegt, obwohl es Zweifel an der geologischen Eignung dieses Standorts gab und sich massiver Widerstand gegen die Standortentscheidung formierte. Die hier untersuchte Endlagersuche auf Grundlage des StandAGs ist das bisherige Ende einer Kette von Kommissionen, Beteiligungs- und Schlichtungsversuchen, die bisher nicht zur Befriedung des Konflikts und der Lösung der Endlagerfrage in Deutschland führten (siehe Kamlage et al. 2019; Losada et al. 2019).

Im Zentrum der vorliegenden Untersuchung steht die erste Phase der Endlagersuche, konkret die bis 2016 tätige „Kommission Lagerung hochradioaktiver Abfallstoffe“ (kurz: Endlagerkommission) und die sie begleitenden Bürgerbeteiligungsverfahren. Da die zweite Phase gerade erst begonnen hat, kann deren Güte derzeit noch nicht beurteilt werden und wird hier nicht untersucht. Es ist allerdings jetzt schon sichtbar, dass auch in Phase 2 ein sehr umfangreiches Spektrum an Beteiligungsformen etabliert wird: Neben dem Nationalen Begleitgremium, das die Öffentlichkeitsbeteiligung am Standortauswahlverfahren begleitet, werden eine Vielzahl formaler, also gesetzlich vorgeschriebener, sowie informeller Beteiligungsformate durchgeführt, die Informations-, Konsultations- und Evaluationsformate umfassen. Zuständig für die Öffentlichkeitsbeteiligung in der zweiten Phase ist das Bundesamt für die Sicherheit der nuklearen Entsorgung (BASE). ${ }^{6}$ Nicht etabliert werden Mechanismen der Öffentlichkeitsbeteiligung, die Entscheidungsgewalt übertragen. ${ }^{7}$

\footnotetext{
5 Zur Geschichte der Endlagersuche in Deutschland siehe etwa Brunnengräber (2016); Losada et al. (2019); aber auch die historische Aufarbeitung durch die Endlagerkommission (Endlagerkommission 2016, insb. S. 77-104) und alternativ die Sicht der Anti-Atom-Bewegung (Bürgerinitiative Umweltschutz Lüchow Dannenberg et al. 2016).

6 Das BASE (bis Ende 2019 unter dem Namen Bundesamt für kerntechnische Entsorgungssicherheit $[\mathrm{BfE}])$ nennt in seinem Beteiligungsansatz dezentral-mobile und zentral am Standort des Bundesamts
} 


\subsection{Die Endlagerkommission}

Auftrag der Endlagerkommission war es, zur Novellierung des StandAGs beizutragen, und zwar erstens durch Einigung auf ein Verfahren zur Endlagersuche, das fair, beteiligend und transparent ist, und zweitens durch die Festlegung auf Kriterien für die Endlagerauswahl. Es ging also noch nicht um die Bestimmung des Standortes selbst. Aufgrund des äußerst hohen Konfliktniveaus und der tiefen Gräben galt eine Einigung auf Kriterien als ein sehr wichtiger erster Schritt, mit dem bereits indirekt der zentrale Konflikt - die Gorlebenfrage - bearbeitet würde.

Die Kommission erhielt Input aus einer Vielzahl an Kanälen der Öffentlichkeitsbeteiligung, die in Abschn. 2.2. genauer untersucht werden. Sie war aber auch selbst ein Beteiligungsinstrument, da sie eine Vielzahl an Gesellschaftsvertretern einschloss. Es handelte sich dabei natürlich nicht um den Versuch der direkten Bürgerbeteiligung, sondern um Stakeholder-Beteiligung, die aber, wie oben gezeigt, keineswegs die grundsätzlich weniger wünschenswerte oder „undemokratischere“ Variante politischer Partizipation ist. Darüber hinaus handelte es sich auch bei der Endlagerkommission um ein deliberatives Forum, das diskursiv und konsensorientiert danach strebt, eine gemeinsam getragene Lösung zu erreichen. Deshalb werden die oben aufgeführten Maßstäbe demokratischer Beteiligung auch an die Kommission selbst angelegt.

\subsubsection{Gleichheitsdimension: Die Inklusivität der Endlagerkommission}

Will man den Gleichheitsaspekt beurteilen, dann muss man zunächst die Frage stellen: Wer ist im vorliegenden Fall vom Problem betroffen? Im Fall der atomaren Endlagerung kommt hier sicherlich die gesamte Bevölkerung in Betracht. Auf kurze Sicht sind die Partikularinteressen von Wirtschaftsunternehmen und Arbeitnehmern der Energiebranche sowie von Bewohnern der Regionen möglicher Standorte stärker betroffen. Auf mittlere und lange Sicht treten gesamtgesellschaftliche Umwelt- und Gesundheitsbelange - insbesondere standortnah - in den Vordergrund sowie die ethische oder moralische Problematik der Belastung nachfolgender Generationen mit hochradioaktivem Müll für eine nicht überschaubare Periode von etwa einer Million Jahre.

verortete Informationsangebote sowie Informationsbroschüren und Online-Angebote insbesondere für die breite Öffentlichkeit und Medien, Gesprächsrunden mit Multiplikatoren, regionale Dialogveranstaltungen, Online-Konsultationen, die Beteiligungswerkstatt Endlagersuche für die junge Generation, Journalistenworkshops, Fachkonferenzen, eine jährliche Statuskonferenz für die engagierte Öffentlichkeit und professionelle Akteure sowie den Austausch mit Beteiligungsexperten (BfE 2019).

7 Das schwedische und das schweizerische Modell, die in der Literatur häufig als Best-Practice-Beispiele gehandelt werden (Kuppler 2016; Seier 2016), sehen solche Mechanismen zwar vor, von der Endlagerkommission und den meisten Bürgerbeteiligungsforen wurden sie aber letztlich nicht begrüßt (Endlagerkommission 2016, S. 433). Insbesondere ein regionales Vetorecht wurde in der Kommission intensiv diskutiert. Ein solches Recht ist in Schweden etabliert, wurde aber vom jetzt ausgewählten Standort Östhammar nicht genutzt, da dort starke Unterstützung für die Standortauswahl herrscht. Zur Erklärung dieser Akzeptanz sei auf einen hochinteressanten Beitrag zur NIMBY-Frage und Freiwilligkeit in der Standortauswahl verwiesen, in dem di Nucci (2016) auf „,nuclear communities“, wie etwa Östhammar, aufmerksam macht, in denen bereits kerntechnische Anlagen stehen und die eine entsprechende ,nukleare Identität“ aufgebaut haben. 
Das StandAG sah vor, dass die Kommission sich zusammensetzt aus: ,acht Vertreterinnen oder Vertretern aus der Wissenschaft, zwei Vertreterinnen oder Vertretern von Umweltverbänden, zwei Vertreterinnen oder Vertretern von Religionsgemeinschaften, zwei Vertreterinnen oder Vertretern aus der Wirtschaft und zwei Vertreterinnen oder Vertretern der Gewerkschaften sowie [...] acht Mitgliedern des Deutschen Bundestages, wobei jede Fraktion im Deutschen Bundestag vertreten ist, und acht Mitgliedern von Landesregierungen“" (StandAG 2013 § 3 Abs. 1). Ausgewählt wurden die Vertreter*innen von Wissenschaft und gesellschaftlichen Interessengruppen auf Grundlage eines gleichlautenden Wahlvorschlags von Bundestag und Bundesrat, ,die Mitglieder des Deutschen Bundestages auf Grundlage eines gemeinsamen Wahlvorschlages von den im Deutschen Bundestag vertretenen Fraktionen und die Mitglieder der Landesregierungen auf Grundlage eines gemeinsamen Wahlvorschlages vom Bundesrat [...]“" (StandAG $2013 \S 3$ Abs. 1).

Die Besetzung der Umweltverbändeposten verzögerte sich, weil sich zunächst keine Umweltorganisation zur Teilnahme bereiterklären wollte. Auch dem atomkritischem .ausgestrahlt e.V. wurde ein Sitz in der Kommission angeboten, den der Verein ablehnte. ${ }^{8}$ Schließlich fand sich zunächst mit der deutschen Umweltstiftung eine kleinere Umweltorganisation, die den ersten Posten besetzte. Im zweiten Schritt entschied sich auch der mitgliederstärkste Umweltverband Deutschlands, der BUND, zur Teilnahme (BUND 2015).

Die Kommission bestand schließlich inklusive zweier Vorsitzender aus vierunddreißig Mitgliedern und wurde durch eine in der Bundestagsverwaltung angesiedelte Geschäftsstelle unterstützt. Den Vorsitz teilten eine CDU-Politikerin und ein SPDPolitiker, die während der Beratungen der Kommission kein Amt oder Mandat innehatten. Den kurzfristigen Interessen von Wirtschaft und Arbeitnehmern in der Energiebranche wurde durch die Einbindung von Gewerkschaften und Energieunternehmen Rechnung getragen und die Interessen möglicher Standorte wurden durch Landesvertreter*innen eingebunden. Umweltorganisationen und christliche Kirchen standen für Kollektivinteressen (Umwelt- und Naturschutz sowie ethische Erwägungen der Lagerung hoch radioaktiven Abfalls). Natürlich kann man sich fragen, weshalb die christlichen Kirchen in einem Land, in dem immer weniger Menschen konfessionell gebunden sind, bei der Endlagersuche eine so entscheidende Rolle einnahmen. Man hätte sich an dieser Stelle auch Jugendorganisationen, Verbraucherschutzgruppen oder humanistische Vereine vorstellen können, aber das StandAG sah die Teilnahme von Religionsgemeinschaften vor.

Da das gesamte im Bundestag vertretene Spektrum Parteienvertreter*innen schickte, war ein Großteil der politischen Positionen der Gesamtbevölkerung abgebildet. Betroffene Perspektiven sind damit in einem relativ hohen Maß abgebildet worden. Nichtsdestotrotz hätte man sich ein stärkeres Gewicht der langfristigen Kollektivinteressen vorstellen können, führt man sich die hohen Risiken und die enorme Dauer der Bedrohung der gesamten Bevölkerung durch den atomaren Müll vor Augen. Darüber hinaus waren Frauen mit nur vier Vertreterinnen stark unterrepräsentiert. Von symbolischer Bedeutung wäre es angesichts der langen

\footnotetext{
${ }^{8}$ Die Zusage knüpfte der Verein an dreizehn Forderungen, die laut eigener Angabe bis auf eine nicht erfüllt wurden, siehe etwa ausgestrahlt 2014.
} 
Problemzeiträume sicherlich auch gewesen, einen Vertreter oder eine Vertreterin der jungen Generation in die Kommission zu berufen. Die Kommission bemühte sich aber, diese Gruppe, ebenso wie die atomkritische Bewegung, über ihre Bürgerbeteiligungskanäle einzubinden (siehe Abschn. 2.2.).

Da die Gesellschaftsvertreter*innen unter den Kommissionsmitgliedern als Stakeholder bestimmter Gesichtspunkte berufen wurden, die also nicht für sich selbst, sondern für eine bestimmte Gruppe stehen, stellt sich in erster Linie die Frage, ob hier glaubwürdige Verantwortlichkeitsbeziehungen bestanden haben, während die Frage der demografischen Ähnlichkeit eher in den Hintergrund tritt. Die Mehrheit der Mitglieder, und zwar die Gewerkschaften, die Parteien und Länderregierungsvertreter*innen, einer der Umweltverbände (BUND) sowie eine*r der Kirchenvertreter*innen standen in klarer Verantwortlichkeitsbeziehung gegenüber ihrer ,,constituency“, waren durch sie direkt autorisiert und ihr gegenüber verantwortlich. Bei einer Minderheit war das Verhältnis weniger eng und direkt: Einer der Kirchenvertreter (Georg Milbradt) scheint mit keiner Kirche in besonderer Weise organisatorisch verbunden zu sein, die Delegierten von Eon und RWE vertraten das Management von zwei privaten Firmen und eine der Umweltorganisationen (Deutsche Umweltstiftung) war keine Mitgliederorganisation, sondern eine Stiftung ohne direkte formale Bindung an eine ,constituency“.9

Gut nachvollziehbar durch die Videoaufnahmen und Interviews mit Kommissionsmitgliedern ist das hohe Maß an Respekt und Fairness in der Kommission. Es wurde begründungsorientiert argumentiert, i.d. R. wurde niemand unterbrochen oder anderweitig marginalisiert, die Vorsitzenden beförderten die offene Debatte und Auseinandersetzung aktiv, indem sie etwa Teilnehmer*innen, die unzufrieden wirkten, gezielt, freundlich und humorvoll dazu aufforderten, ihren Unmut offen zu äußern, und so fanden alle Stimmen Gehör. Selbst mit den beiden schärfsten Konflikten wurde ein Umgang gefunden, indem die Entscheidung über die Finanzierung der Endlagerung in eine kleinere Extrakommission ausgelagert wurde und man sich auf Kriterien einigte, die die Auswahl des Standorts Gorleben im Grunde ausschlossen. Tatsächlich war Gorleben nicht unter den über 90 möglichen Regionen, welche auf Basis der in der Kommission entwickelten Kriterien im September 2020 von der Bundesgesellschaft für Endlagerung als mögliche Standorte vorgestellt wurden.

\subsubsection{Wirkungsdimension: Die Resonanz der Kommissionsvorschläge}

Die Endlagerkommission bemühte sich, im Einklang mit dem StandAG ( 1 Abs. 1) um möglichst weitgehende Einigung, versuchte Abstimmungen zu vermeiden und verankerte das Konsensziel in ihrer Geschäftsordnung ( 3 Geschäftsordnung der Kommission Lagerung hochradioaktiver Abfälle). Nichtsdestotrotz wurde über alle umstrittenen Teilabschnitte des Ergebnisberichts nach und nach mit Zweidrittelmehrheit abgestimmt. An diesen Abstimmungen nahmen alle Mitglieder teil. An der Endabstimmung über den gesamten Bericht stimmten indes nur die als Wis-

\footnotetext{
9 Allerdings engagiert sich diese Stiftung als Non-Profit-NGO seit Jahrzehnten umweltpolitisch in Deutschland und wird von Medien und anderen Gruppen bei ihren Tätigkeiten beobachtet, wodurch sie in einer indirekten Verantwortlichkeitsbeziehung steht (siehe Urbinati und Warren 2008, S. 405).
} 
senschaftler und Interessengruppen berufenen 16 Kommissionsmitglieder ab, da nur diese laut Geschäftsordnung Stimmrecht über den Endbericht hatten ( $\$ 7$ Abs. 2 Geschäftsordnung der Kommission Lagerung hoch radioaktiver Abfälle). Der de facto ausschließende Effekt dieser Regelung war allerdings gering, da zum Zeitpunkt der Endabstimmung die strittigen Fragen geklärt waren und es lediglich um eine kollektive Anerkennung des Berichts durch ,Nostrifizierung ' ging. ${ }^{10}$ Abweichende Meinungen in Form von Sondervoten zu einzelnen Fragen waren erlaubt und haben die umfassende Einigung auf das Endergebnis sicherlich erleichtert.

Dem Endergebnis stimmte schließlich ein Mitglied, der Vertreter des BUND, nicht zu (Endlagerkommission 2016, S. 497), wenngleich er in der Kommission durchgehend konstruktiv mitgearbeitet und den Bericht aktiv gestaltet hatte. Die Kommission selbst bezeichnet ihr Aggregationsergebnis dennoch als „,breiten Konsens“, als ,überwältigende Mehrheit“" und als „Einvernehmen“ (siehe Endlagerkommission 2016, S. 27, 30), mit dem das Konsensziel erreicht worden sei.

Trotz der Sondervoten von sechs Mitgliedern zu einzelnen Fragen und der Gegenstimme eines Mitglieds zum Gesamtbericht integrierte die Kommission einen Großteil politischer und gesellschaftlicher Gruppen und Perspektiven zu einer gemeinsamen Stimme. Wenngleich dieser Konsens keineswegs als breite, allumfassende Zustimmung aller Betroffenen und Interessierten gelten kann und deshalb nicht dauerhaft stabil sein muss (Krick 2013, 2017), ist das Ergebnis doch bemerkenswert, wenn man die tiefen und jahrzehntealten ideologischen Gräben rund um das atomare Endlager in Deutschland bedenkt sowie die vielen gescheiterten Versuche, in dieser Frage politisch weiterzukommen. Das Ergebnis der Kommission zeichnet sich dadurch aus, dass es politische Spektren überbrückt. Insbesondere überwindet es die Kluft zwischen Gorleben-Gegnern und Gorleben-Befürwortern und involviert die Grünen, die lange das parteipolitische Sprachrohr der Anti-Atombewegung waren. Nichtsdestotrotz vermochte es nicht, die dezentralen und vielgestaltigen zivilgesellschaftlichen Gruppen, die sich seit Jahrzehnten kritisch mit der der nuklearen Entsorgung befassen, vom Beteiligungsprozess oder Kommissionsergebnis zu überzeugen und auch der BUND sowie die Partei die LINKE äußern sich dem Beteiligungsprozess gegenüber heute vorwiegend kritisch.

Die Kommission entwarf gemäß ihres Auftrags detaillierte Kriterien für die Standortauswahl und machte ausführliche Vorschläge zur Reform der Verwaltungsstruktur und Ausgestaltung der Beteiligungsverfahren in der zweiten Phase. Sie fügte dem Bericht außerdem eine historische Aufarbeitung des Endlagerkonflikts und eine ethische Reflexion des Endlagerproblems hinzu. ${ }^{11}$ Die Vorschläge können als relevant, problemadressierend und konkret bezeichnet werden. Durch die Teilnehmerstruktur waren sie mit allen politischen Parteien des Bundestags abgestimmt. Auch Vertreter*innen der Bundesministerien sorgten für Rückkopplung. Sie nahmen als ständige Beobachter an den Sitzungen der Kommission teil, beteiligten sich aber nicht aktiv an

\footnotetext{
10 Durch einen Akt der Nostrifizierung nimmt eine Gruppe einen Bericht oder eine Entscheidung formal als ihre Stimme, ihre gemeinsame Sichtweise an. Minderheitenvoten stehen dieser Praktik nicht notwendig entgegen (Lentsch und Weingart 2011, S. 367).

11 Sie fügte dem Bericht außerdem eine historische Aufarbeitung des Endlagerkonflikts und eine ethische Reflexion des Endlagerproblems hinzu.
} 
den Verhandlungen. Auch die parlamentarische Debatte des StandAGs macht nachvollziehbar, wie mit den Kommissionsvorschlägen umgegangen wurde und welche Elemente des Berichts als Ergebnis der politischen Auseinandersetzung noch angepasst wurden. Transparenz und Rückkopplung wurden auch durch die Doppelrolle der Abgeordneten in der Kommission hergestellt, die sowohl die Kommissionsvorschläge mit erarbeitet hatten als auch Sitze im zuständigen Umweltausschuss innehatten und daher intensiv in die Gesetzgebung eingebunden waren. Sie stellten die Beziehung zwischen Kommissionsvorschlägen und Gesetz her und kommentierten dies öffentlich.

Der Kommissionsbericht wurde offiziell in den politischen Prozess eingespeist, als er am 05.07.2016 dem Bundestagspräsidenten übergeben wurde. Der Bundestag nahm den Kommissionsbericht einstimmig zur Kenntnis. Aufbauend auf dem Kommissionsbericht und einem Kabinettsentwurf wurde ein Entwurf eines Gesetzes zur Änderung des StandAGs von allen im Bundestag vertretenen Parteien in den Bundestag eingebracht und im Umweltausschuss behandelt. Laut verschiedener Bundestagsfraktionen wurden die Vorschläge der Kommission zu den Verfahren und Kriterien der Standortsuche „1:1“ in das reformierte StandAG übernommen (BundestagsDrucksache 18/11647, 15; 18/11648, Bundestags-Plenarprotokoll 18/179, 181), entwickelten also ausgesprochen starke Policy-Wirkung. Es gab freilich geringfügige Abweichungen vom Kommissionsbericht, wovon einige infolge einer Anhörung von Interessengruppen vor dem zuständigen parlamentarischen Ausschuss (Umweltausschuss) aufgenommen worden waren. ${ }^{12}$ Der Gesetzentwurf wurde parteiübergreifend angenommen und die Zustimmung ging damit über die Regierungsmehrheit hinaus, da nur die Partei Die Linke dagegen stimmte. „Soviel Einigkeit findet sich selten in diesem Hohen Haus“, bemerkte ein Abgeordneter der CDU/CSU während der Beratungen des Gesetzes zur Änderung des StandAGs (Bundestags-Plenarprotokoll 18/179, 181). Neben dieser enormen direkten Wirkung auf die Gesetzgebung fanden die Verhandlungen der Kommission (und ihrer Bürgerbeteiligungskanäle) auch im politischen Diskurs ein Echo, vor allem innerhalb der interessierten Öffentlichkeit und der parlamentarischen Debatte.

\subsection{Die Bürgerbeteiligung in der ersten Phase der Endlagersuche}

Die Endlagerkommission beauftragte zu Beginn ihrer Arbeit im Frühjahr 2014 die Beratungsfirma Demos damit, ein Konzept zur Bürgerbeteiligung zu entwerfen, das Demos mithilfe weiterer Beratungsfirmen (e-fekt, Prognos AG, zebralog) umsetzte (Demos/Prognos 2015). Die Endlagerkommission bekam in der Folge Input aus einer Fülle an unterschiedlichen Beteiligungsformaten (siehe Tab. 2, die aus dem Kommissionsbericht stammt und die Formate im Überblick zeigt).

\footnotetext{
12 Die geringfügigen Abweichungen des Gesetzes gegenüber den Vorschlägen im Kommissionsbericht betrafen beispielsweise Details der Gestaltung der Bürgerbeteiligung in der zweiten Phase und der Verwaltungsstruktur, die für den Auswahlprozess zuständig ist. Veränderungen betrafen auch einzelne Formulierungen. So ist aus dem Gesetzentwurf gestrichen worden, dass es in erster Linie um die Auswahl eines Standorts für hoch radioaktiven Müll geht (https://www.bundestag.de/dokumente/textarchiv/2017/kw12de-atommuell-standortauswahl/496742).
} 
Tab. 2 Bürgerbeteiligungsformate der Endlagerkommission (erste. Phase der Endlagersuche). (Quelle: Endlagerkommission 2016, S. 413)

\begin{tabular}{|c|c|c|}
\hline Beteiligungsformat & Formate & Status \\
\hline Bürgerdialog Standortsuche & Plenarveranstaltung mit Arbeitsgruppen & Durchgeführt \\
\hline Informationskampagne & $\begin{array}{l}\text { Information der breiten Öffentlichkeit } \\
\text { über Kerninhalte des Entwurfs }\end{array}$ & Zurückgestellt \\
\hline Workshops mit den Regionen & Drei Workshops (je eintägig) & Durchgeführt \\
\hline $\begin{array}{l}\text { Workspace und Fachkonferenz mit der } \\
\text { Fachöffentlichkeit }\end{array}$ & Online-Workspace und Fachkonferenz & Durchgeführt \\
\hline $\begin{array}{l}\text { Junge Erwachsene und Beteiligungs- } \\
\text { praktiker }\end{array}$ & Drei Workshops (je zweitägig) & Durchgeführt \\
\hline Dialogangebote kritische Gruppen & Dokumentenanalyse & Durchgeführt \\
\hline Zuschriften und Online-Format & Kommentierung des Entwurfs im Web & Durchgeführt \\
\hline Kommissionsbericht im Entwurf & $\begin{array}{l}\text { World Café mit vernetzter Dokumentati- } \\
\text { on und Plenarphasen }\end{array}$ & Durchgeführt \\
\hline
\end{tabular}

Hinter dem Eintrag „Zuschriften und Online-Format“ in Tabelle 2 verbergen sich verschiedene Inputkanäle und Deliberationsforen. Es gab die Möglichkeit, E-Mails an die Geschäftsstelle oder Zuschriften über den Online-Auftritt zu senden sowie ein Internetdiskussionsforum und eine Online-Diskussion des Berichtsentwurfs zu nutzen. Über diese, in Tabelle 2 gelisteten, offiziellen Beteiligungsformate hinaus gab es weitere, unabhängige Formate, wie etwa das ENTRIA-Bürgergutachten, die nicht Teil des Kommissionskonzeptes waren, aber in den Empfehlungen der Kommission berücksichtigt wurden (Endlagerkommission 2016, S. 431). Während des Prozesses wurde die Bürgerbeteiligung zudem durch zwei auf Beteiligungsfragen spezialisierte Institutionen der angewandten Forschung und Beratung evaluiert (Dialogik gemeinnützige GmbH und European Institute for Public Participation [EIPP]) (siehe auch Dialogik/EIPP 2016).

\subsubsection{Gleichheitsdimension: Die Inklusivität der Bürgerbeteiligung}

Wie bereits beschrieben, ist der Kreis der vom Thema Betroffenen groß, wobei einzelne Zielgruppen besonders direkt oder unmittelbar betroffen sind und daher besonderer Berücksichtigung bedürfen. Das Beteiligungskonzept der Kommission berücksichtigt dies und nennt die folgenden zentralen Zielgruppen der Bürgerbeteiligungsformate: die ,breite“ oder ,,allgemeine“ Öffentlichkeit (d.h. nichtprofessionell mit dem Thema befasste, ,normale“ Bürger), die engagierte Öffentlichkeit (d.h. sich für das Thema engagierende und entsprechend fachkundige, aber nichtprofessionelle Akteure), die Fachöffentlichkeit, junge Menschen und Beteiligungspraktiker (u. a. aus den Kommunen), Regionenvertreter*innen möglicher Standorte sowie die „kritische“ Öffentlichkeit (d.h. die Anti-Atom-Bewegung). Die verschiedenen Beteiligungsformate waren jeweils für unterschiedliche Cluster dieser Teilöffentlichkeiten konzipiert (Demos/Prognos 2016, S. 23-25). ${ }^{13}$

${ }^{13}$ So sollte etwa die breite Öffentlichkeit durch den Bürgerdialog, die Informationskampagne, die Angebote an die kritische Öffentlichkeit, Zuschriften und Online-Formate sowie die Veranstaltung „Kon- 
Die Auswahl der Teilnehmer erfolgte je nach Format unterschiedlich: So wurden für die Workshops mit Jugendlichen etwa Studienwerke deutschlandweit, Schulen in Umkreis Dortmund und Jugendorganisationen angeschrieben, woraufhin sich junge Menschen um die Teilnahme bewarben und aus diesen (nach demografischen Kriterien der statistischen Repräsentation) ausgewählt wurden (Dialogik/EIPP 2016, S. 33). Zur Fachtagung wurden Einladungen an ,Expertinnen und Experten aus den Bereichen Endlagerung, Geowissenschaften, Bergbau und Planungswissenschaften, aus Universitäten, Forschungseinrichtungen, Bundes- und Landesämtern, Bürgerinitiativen, Verbänden und Unternehmen“ über einen Verteiler der Kommission gesandt. Darüber hinaus wurde eine Recherche zu atomrelevanten NGOs durchgeführt und diese angeschrieben (EIPP/Dialogik 2016, S. 45). Unter den 200 Teilnehmenden waren dann nicht nur zahlreiche Wissenschaftler, sondern auch Vertreter*innen von Politik und Verwaltung und der Energiewirtschaft, engagierte Bürger*innen, Vertreter*innen von Bürgerinitiativen und Umweltgruppen und der Anti-Atom-Bewegung (EIPP/Dialogik 2016, S. 46). Zu den Regionenworkshops wurden die betreffenden Regionenverwaltungen angeschrieben und zur Auswahl und Entsendung von Vertretern gebeten. Zu den Onlineforen war der Zugang dagegen offen, was laut Beteiligungskonzept und Evaluationsbericht vor allem Interessengruppen zur Darstellung konträrer Positionen nutzten. ${ }^{14}$

In der Evaluation der Institute Dialogik und EIPP wird die mehr oder weniger ausgeprägte Verzerrung der Bürgerbeteiligungsformate $\mathrm{zu}$ Ungunsten von jüngeren Personen, Frauen, ökonomisch schlechter gestellten und nichtprofessionellen ,normalen“ Bürger*innen angemerkt (Dialogik/EIPP 2016, S. 67). Es wird bemängelt, dass vor allem durch die frühen Formate in erster Linie Fachpublikum sowie Vertreter*innen von (regionalen) Behörden und Verwaltungen angezogen wurden, sodass die Evaluation zu dem Urteil kommt: ,Einen auch nur annähernd repräsentativen Querschnitt der Bevölkerung konnten die Veranstaltungen nicht abbilden“ (Dialogik/ EIPP 2016, S. 67). Empfohlen wird zur faireren Selektion in Zukunft die Zufallsauswahl (Dialogik/EIPP 2016, S. 3).

Allerdings steuerte die Kommission hier erstens nach, indem sie drei spezielle Workshops mit jungen Erwachsenen veranstaltete und die Anti-Atom-Bewegung integrierte, die kaum an den Formaten der Bürgerbeteiligung teilnahm und dem Prozess gegenüber grundsätzlich kritisch eingestellt blieb. ${ }^{15}$ Durch eine Dokumen-

sultation zum Berichtsentwurf“ angesprochen werden, während die Regionenvertreter an den Regionenworkshops, der Konsultation des Berichtsentwurfs sowie den Zuschriften und Online-Formaten teilnehmen sollten.

14 Es gab zwei verschiedene Online-Foren. Im Internetdiskussionsforum, das nach etwa 10 Monaten Kommissionslaufzeit freigeschaltet wurde, gab es starken Unmut über die Moderation und verzögerte Freischaltung der Beiträge. Als die Moderation der Beiträge beendet wurde, übernahmen Trolle die Foren und eine sinnvolle Diskussion war nicht mehr möglich. Daraufhin und als Ergebnis der Zwischenevaluierung ihres Beteiligungsansatzes wurde eine Online-Konsultation des Berichtsentwurfs etabliert, die lebhaft und sachbezogen war. Deren Input wurde in der Kommission und seinen AGs intensiv bearbeitet und das Format wird auch in der Evaluation als gelungen beschrieben (Endlagerkommission 2016, S. 418). Mit den Onlinekommentaren zu besonders diskutierten Berichtsteilen (etwa der Teil „Zum Umgang mit Konflikten“) setzte sich die zuständige AG der Kommission auseinander.

15 Die Bürgerinitiativen und kleinen, oft regionalen NGOs der Anti-Atom-Bewegung bemängelten u.a., dass das StandAG der Kommissionseinsetzung voraus ging, dass nur hoch radioaktiver Müll im Zentrum 
tenanalyse der Online-Statements der Bewegung wurden deren Positionen in die Kommissionsdebatte einbezogen. Außerdem nahmen mehrere Vertreter der AntiAtom-Bewegung an der Fachkonferenz teil. Die Positionen der Bewegung wurden darüber hinaus in der Kommission auch von der Partei die Linke eingebracht sowie vom Vertreter des Landes Niedersachsens, in welchem der umstrittene Gorlebenstandort liegt.

Zweitens war ein repräsentativer Querschnitt der Bevölkerung gar nicht das Ziel des Beteiligungsansatzes gewesen. Vorgesehen waren vielmehr die Ansprache bestimmter Zielgruppen, das Anstoßen einer öffentlichen Debatte und die Involvierung möglichst vieler Menschen (Demos/Prognos 2015, S. 5). Hätte man eine demografische Ähnlichkeit der Ausgewählten mit der Gesamtbevölkerung erreichen wollen, hätte man vor allem auf Zufallsstichproben setzen müssen. Das war jedoch explizit nicht angestrebt (siehe bspw. Demos/Prognos 2015, S. 38).

Drittens löst die Zufallsauswahl (oder auch Quotenstichprobe), die der Evaluationsbericht empfiehlt und die heute oft als die Antwort auf Repräsentativitätsprobleme gehandelt wird, keineswegs alle Schwierigkeiten der Bürgerbeteiligung. Da Zufallsstichproben kaum durch Zwang durchgesetzt werden können, sind sie enorm ressourcenaufwändig, wenn die Auserwählten für die Teilnahme angemessen entschädigt werden sollen. Auch können die durch Zufalls- oder Quotenstichproben Ausgewählten bei der begrenzten Teilnehmerzahl von deliberativen Foren nur in Bezug auf eine Handvoll (demografischer) Kriterien die Bevölkerung abbilden, also beispielsweise Geschlecht, Alter und Bildungsstand aber keineswegs in Bezug auf alle relevanten Merkmale (Krick 2019). Außerdem zieht die Stichprobenauswahl, ähnlich wie der offene Zugang, natürlich epistemische Fragen in Bezug auf Kompetenz und Engagement der Auserwählten nach sich.

Viertens ist die deskriptive Ähnlichkeit oder statistische Repräsentativität nicht der einzige gültige Repräsentationsstandard. Er konkurriert insbesondere mit der substanziellen Repräsentation, die durch die Involvierung betroffener Perspektiven durch legitimierte Stellvertreter erreicht wird. Wenn man sich die Beteiligtenstruktur insgesamt ansieht, dann ist die Beteiligung der Zielgruppen, die für besonders betroffene Interessen und Perspektiven stehen, durch Stellvertreter durchaus gelungen. Nicht immer standen diese Stellvertreter in direkten Verantwortlichkeitsbeziehungen, wie dies etwa bei Mitgliederorganisationen der Fall ist. Nicht alle Vertreter der Jugend beispielsweise waren durch eine Jugendorganisation entsandt. Einige qualifizierten sich einfach, weil sie der Zielgruppe durch ihr Alter grundsätzlich angehörten, durch eine Bewerbung Interesse am Thema gezeigt hatten und zur Diversität beitrugen. Ebenso wenig konnte eine Balance zwischen antagonistischen Interessen hergestellt werden. Als Gegengewicht zu den Interessen der Energiewirtschaft waren allerdings stets Vertreter von Kollektivinteressen, insbesondere der Umweltorganisationen anwesend (Interview E; siehe auch Demos/Prognos 2016, S. 45). Vertreter von Vereinen und Initiativen der kritischen Öffentlichkeit waren indirekt durch die Dokumentenanalyse, aber auch die Teilnahme an der Fachveranstaltung beteiligt. Insgesamt darf man sagen, dass die besonders betroffenen Interessen weitgehend,

der Kommissionsarbeit stand und dass Alternativen zur tiefengeologischen Lagerung nicht genug Beachtung fanden (Bürgerinitiative Umweltschutz Lüchow Dannenberg et al. 2016, S. 5-7, 25-38). 
durch mehr oder weniger direkt legitimierte Stellevertreter vertreten waren, sodass das Kriterium der substanziellen Repräsentation in hohem Maße erfüllt wurde.

Der zentrale Rekrutierungs- und Zugangsweg zu den Beteiligungsformaten war die Einladung durch die Veranstalter. Dieser Mechanismus der gezielten Auswahl oder bewussten Zielgruppenansprache hat seine Stärken, wenn es um die Ansprache bestimmter Stakeholder geht. Weniger wurde einzig die Zielgruppe der ,,breiten Öffentlichkeit" erreicht. Die dieser Zielgruppe zugeordneten Personen unterscheiden sich wohl von den als ,engagierte“, ,interessierte“ oder „kritische“ Öffentlichkeit oder als „Fachpublikum“ bezeichneten Zielgruppen dadurch, dass sie nicht-organisiert ist und sich weder privat bereits für das Thema interessieren noch professionell damit befasst sind. Um diese Gruppe stärker einzubinden, hätte es möglicherweise geholfen, die ursprünglich geplante Informationskampagne durchzuführen und mit Anreizstrukturen (wie Reisekosten oder Entschädigungen) oder auch Quotenstichproben zu kombinieren.

In Befragungen, die dem Evaluationsbericht zugrunde liegen, wird von den Teilnehmenden die angenehme Atmosphäre in den Beteiligungsforen betont, die hohe Diskussionsqualität und der respektvolle Umgang miteinander (Dialogik/EIPP 2016, S. 8-53). Die Moderation der Veranstaltungen wird in der Evaluation ebenfalls durchweg sehr gelobt und als fair, sachorientiert, unparteiisch, nichtsteuernd und kompetent beschrieben. Man gewinnt den Eindruck, dass der Deliberationsprozess insgesamt inklusiv war und keine informellen Ausschlussmechanismen wirkten. Nur der Zeitmangel wurde wiederholt als hinderlich für intensive Debatten kritisiert (Dialogik/EIPP 2016, S. 68-69; Endlagerkommission 2016). ${ }^{16}$

\subsubsection{Wirkungsdimension: Die Resonanz des Bürgerinputs}

Die Deliberationen in den einzelnen Bürgerforen waren konsensorientiert. Es sollten keine Mehrheitsabstimmungen stattfinden und ein Vorschlag nur als Konsens festgehalten werden, wenn alle dafür waren (Demos/Prognos 2016, S. 13). Mit der Schwierigkeit der Vielstimmigkeit, die sich ergibt, wenn etwa 20 verschiedene Inputkanäle geöffnet werden und kein Aggregationsmechanismus vorgesehen ist, der diese Stimmen zu klaren Botschaften an die Entscheidungsverantwortlichen (oder sogar einem Konsensergebnis) zusammenführt, wurde folgendermaßen umgegangen: Der Input aus den verschiedenen Beteiligungsforen wurde durch die Beratungsfirmen Demos und Prognos in Ergebnisprotokollen gesammelt, verdichtet und in Zusammenarbeit mit der Geschäftsstelle der Kommission in online zugänglichen Tabellen aufbereitet.

\footnotetext{
16 Der Evaluationsbericht scheint zu einem kritischeren Ergebnis zu kommen als die vorliegende Analyse. Dafür gibt es eine Reihe von Gründen: Die Evaluation war prozessbegleitend konzipiert, um Nachsteuerung während des Verfahrens zu erlauben und sie wurde entsprechend bereits vor Ende des Beteiligungsprozesses abgeschlossen. Der Evaluationsbericht kann daher nicht die Nachjustierungen mit einbeziehen, die auf seiner Grundlage vorgenommen wurden. Darüber hinaus bezieht sich die Evaluation nur auf die Ebene der Bürgerbeteiligungsformate, während hier die gesamte Mehrebenenstruktur in den Blick genommen wird. Es wird darüber hinaus hier nicht nur die Inklusivität des Prozesses, sondern zweitens die Wirkungsdimension der Beteiligung analysiert. Und schließlich legt der Evaluationsbericht den repräsentativen Querschnitt der Bevölkerung als zentralen Maßstab zugrunde, während hier verschiedene Repräsentations- und Selektionsmechanismen als legitim betrachtet werden.
} 
Diese Tabellen dienten als funktionstüchtiger Aggregationsmechanismus. Sie stellen auf fast 200 Seiten den Inhalt der einzelnen Vorschläge aus den Beteiligungsforen, den Zustimmungsgrad, die Unterstützer des Vorschlags und die Verarbeitung durch die Endlagerkommission dar. Hier ist auch ersichtlich, wie stark die Zustimmung jeweils war und aus wie vielen verschiedenen Foren ein bestimmter Anstoß kam.

Diese Tabellen dienten der Kommission als Informations- und Entscheidungsgrundlage. Sie ermöglichten aber auch der Öffentlichkeit die Beobachtung des Beteiligungsprozesses und machten nachvollziehbar, welchen Einfluss die Bürgervorschläge im Einzelnen entwickelten. Zusätzlich zu diesem Dokument gab es weitere Rückkopplungskanäle zwischen der Kommission und den Beteiligungsformaten, über die sich der Einfluss nachvollziehen lässt. Diese dürften die Einflusschancen erhöht haben, weil die Kommission über diese Kanäle auf den Bürgerinput vorbereitet war: In den Dialogforen waren beispielsweise immer Kommissionsmitglieder als Botschafter zugegen, die für die Rückkopplung zuständig waren, die Arbeit der Kommission also erklärten und die Sicht der Bürger*innen in die Kommission trugen. In den Online-Foren moderierte die Geschäftsstelle der Kommission, aber auch ein Kommissionsmitglied bemühte sich regelmäßig dort um Erläuterungen der Kommissionsarbeit. Vertreter der Jugendforen wurden in die Kommission zur Berichterstattung eingeladen und ein Vertreter der Geschäftsstelle berichtete in allen Sitzungen der Kommission zu Beginn über neueste Anregungen aus Online-Forum und Briefzuschriften. Die Kommission beschäftigte sich mit jeder einzelnen dieser Eingaben, was in den online zugänglichen Videos der Sitzungen nachzuvollziehen ist. Den Umgang mit dem Bürgerinput, also auch die Ablehnung und Nichtaufnahme bestimmter Vorschläge, rechtfertigte die Kommission ausführlich in ihrem Abschlussbericht (Endlagerkommission 2016, insbesondere S. 422-441). Darüber hinaus sind alle Diskussionen, auch die des Bürgerinputs in der Kommission, öffentlich nachvollziehbar, da alle Sitzungen sowohl als Videoaufnahmen als auch als Wortprotokolle auf der Webseite der Endlagerkommission abrufbar waren. Insgesamt lässt sich daher sagen, dass die beteiligten Bürger die Chance hatten, die Kommissionsarbeit zu beeinflussen und damit auch auf das StandAG, das zentrale Objekt der Kommissionsberatungen, einzuwirken.

Die erarbeiteten Vorschläge waren im Großen und Ganzen policy-relevant und konkret, allerdings mit Abstufungen. Sie entwickelten deutliche Resonanz in der Kommission und einige der besonders konkreten Maßnahmen aus den Bürgerforen wurden direkt in den Kommissionsbericht übernommen. Beispiele dafür sind die Vorschläge zur Konkretisierung der Aufgaben des Nationalen Begleitgremiums, das den weiteren Endlagersuchprozess beobachten soll, oder zu möglichen Kompensationen für potenzielle Standorte (Endlagerkommission 2016, S. 433).

Es gab außerdem viele allgemeinere Anregungen der Dialogforen, die in der Kommission diskutiert und aufgenommen wurden, vor allem zur Ausgestaltung des weiteren Suchverfahrens und zur Form der Beteiligung, weniger etwa zu physikalischen Kriterien der Endlagerung. Allerdings war keine verbindliche Entscheidungsmacht auf die Bürgerforen übertragen worden, was von der ,kritischen Öffentlichkeit“" und 
auch im Evaluationsprozess immer wieder bemängelt wurde. ${ }^{17}$ Wie in Abschn. 1 ausgeführt, ist die Übertragung von Entscheidungsmacht auf partizipative Foren mit begrenzter Repräsentativität allerdings demokratietheoretisch nicht unproblematisch. Außerdem gab es keinen Konsens der Beteiligungsforen, keine klare Botschaft, die die Kommission hätte übernehmen können, da die Ergebnisse der verschiedenen Formate teilweise sehr unterschiedlich waren (Endlagerkommission 2016, S. 433). So befürworteten etwa der Bürgerdialog und die Workshops der Regionen verschiedene Formen des Nachteilsausgleichs für potenzielle Standorte, während die kritischen Gruppen Anreize als Bestechung sahen. Ähnlich gingen die Meinungen zum Nationalen Begleitgremium auseinander, in die die „Workshops Junge Menschen“ vor allem ,Zufallsbürger“ entsenden wollte, während die Regionenworkshops eher auf die Pluralität wissenschaftlicher Disziplinen setzten.

\section{Schlussfolgerungen: Die demokratische Qualität der Endlagersuche}

Schaut man sich die demokratische Qualität des Beteiligungsprozesses in der Gesamtschau an, dann lässt sich sagen, dass sowohl die Endlagerkommission als auch die Bürgerbeteiligungsarenen, aber vor allem der Prozess in seiner Gesamtheit die Kriterien der Inklusivität und der Resonanz weitreichend erfüllen. Dieses Ergebnis soll im Folgenden kurz und systematisch zusammengefasst werden. Gleichzeitig sollen die Mechanismen, die zum Erfolg beigetragen haben, verdeutlicht und weiterführende Schlussfolgerungen gezogen werden für die Beteiligungstheorie und -praxis in Deutschland. Besondere Aufmerksamkeit gilt den Aspekten der Analyse, die demokratie- und institutionentheoretische Aufschlüsse erlauben.

Für die Gleichheits- und Inklusivitätsdimension lässt sich zunächst festhalten, dass beide Ebenen dem Standard der authentischen, fairen und inklusiven Deliberation genügten. Hervorzuheben sind die geschickt agierenden, ausgleichenden Moderatoren bzw. Kommissionsvorsitzenden, die Wert darauf legten, Ausschlusseffekte während der Interaktion zu minimieren. Sowohl die Endlagerkommission als auch die Bürgerbeteiligungsforen erfüllten außerdem den Standard der substanziellen Repräsentativität, der auf legitimer Stellvertretung der vom Thema betroffenen Interessen und Perspektiven beruht.

Obwohl man sich auf beiden Ebenen ein noch größeres Gewicht der Kollektivinteressen, oder beispielsweise von Frauen und sozial weniger privilegierten Personen sowie eine noch direktere Autorisierung durch die jeweilige „,constituency“, hätte wünschen können, waren insgesamt doch alle betroffenen Perspektiven vertreten, wobei ein Großteil der Repräsentanten in mehr oder weniger direkten Verantwortlichkeitsbeziehungen stand. Das relativ hohe Inklusivitätsniveau ist auch der ehrgeizigen Zielsetzung und der komplexen Mehrebenenstruktur geschuldet. Durch die Vielfalt der Arenen wurde ein sehr großer Kreis an Zielgruppen involviert. Auf bei-

\footnotetext{
17 Die ,kritische Öffentlichkeit“ bemängelte vor allem, dass einzelne Vorschläge aus den Bürgerforen nicht die erwünschte Durchschlagskraft entwickelten und dass der Prozess aufgrund seiner strukturellen Komplexität und der Menge an verfügbaren Informationen unübersichtlich gewesen sei (siehe bspw. Bürgerinitiative Umweltschutz Lüchow Dannenberg 2016; endlagerdialog.de 2018; Kostka 2018).
} 
den Ebenen wurden Varianten der zielgerichteten Teilnehmerauswahl (oder bewussten Zielgruppenansprache) angewandt, wobei der Zugang zu den Bürgerforen vor allem durch Einladung und Selbstselektion auf dieser Grundlage erfolgte, während die Kommission reservierte Plätze für bestimmte Zielgruppen besetzte. Es wurden auf diese Weise alle anvisierten Zielgruppen, inklusive junger Erwachsener und der ,interessierten Öffentlichkeit“, involviert und selbst für die Positionen der ,kritischen Öffentlichkeit" (also der Anti-Atom-Bewegung) fanden sich Zugangswege. Einzig die Zielgruppe der ,breiten Öffentlichkeit“ wurde durch diese Einladepraxis kaum erreicht. Für sie wäre möglicherweise die Zufallsauswahl, mit der man deskriptive Ähnlichkeit in Bezug auf demografische Kriterien herstellen kann, geeigneter gewesen. Auf diese Weise hätte man den Überhang an männlichen, gut ausgebildeten Teilnehmern ausgleichen können.

Allerdings war auch niemandem aus der ,breiten Öffentlichkeit“ der Zugang verwehrt. Es stellt sich außerdem generell die Frage, wer überhaupt zu dieser Zielgruppe gehören mag und ob die aktive Teilnahme einer solchen Gruppe nicht eine Fiktion ist. Denn schließlich dürfte eine Person, die sich für eine Teilnahme an Bürgerforen zur Endlagersuche entscheidet, automatisch der ebenfalls im Prozess adressierten Zielgruppe der ,,interessierten“ oder ,,engagierten“ Öffentlichkeit zugerechnet werden, wodurch eine Beteiligung der ,,breiten Öffentlichkeit“ letztlich unerreichbar wäre.

Die Analyse unterstreicht, dass die soziale Verzerrung vor allem aus der Perspektive der deskriptiven, statistischen Repräsentation ein Problem ist, aus der Perspektive der substanziellen Repräsentation aber eher in den Hintergrund tritt. Sie zeigt auch, dass die verbreitete Höherbewertung von statistischer Repräsentativität und Zufallsauswahl leicht die Schwächen dieses Selektionsmechanismus übersieht. Dazu zählen etwa die hohen Kosten von Anreizen, die nötig sind, um soziale Verzerrungen auszugleichen. Diese beiden Aspekte, der Hang zu Zufallsauswahl und deskriptiver Ähnlichkeit, aber auch die Konstruktion einer breiten, d.h. nichtorganisierten, nichtprofessionellen Öffentlichkeit als besonders wichtige Zielgruppe, kennzeichnen weite Teil der Beteiligungsdebatte in Deutschland, und sie sind nicht unproblematisch. Sie können einem negativen Bias gegenüber Beteiligungsprozessen Vorschub leisten, zur Aufstellung unerreichbarer Standards beitragen und Enttäuschungen vorprogrammieren.

Vor diesem Hintergrund legen die Ergebnisse der Studie eine Anerkennung der Vielfalt von Repräsentationsprinzipien und eine Neubewertung von Selektionsmechanismen nahe. Sie sprechen für die Rehabilitierung der substanziellen, assoziativen Repräsentation und für das Erwägen eines in der Deliberations- und Partizipationsforschung eher unterschätzten Auswahlmechanismus, also der zielgruppenorientierten, kriteriengeleiteten Teilnehmerselektion unter Anwendung von Praktiken wie Zielgruppenrecherche, bewusste Einladung, Bewerbungsverfahren etc. Eine solche Auswahl birgt die Chance der relativ ressourcenschonenden Herstellung von substanzieller Repräsentation, sie ist aber auch voraussetzungsvoll. Es liegt eine große Macht in der Teilnehmerauswahl und diese ist beim Parlament sicher besser aufgehoben als bei externen Dienstleistern, die einen Beteiligungsansatz konzipieren. Außerdem sollte angestrebt werden, Kriterien und Prozess der Auswahl so transparent wie möglich zu halten und unter Einbeziehung der Öffentlichkeit kritisch 
zu diskutieren. Dies betrifft nicht nur die Zielgruppendefinition und die Festlegung demografischer Kriterien für die Auswahl, sondern auch Prozessschritte wie die Zusammenstellung eines Einladungsverteilers oder die Auswertung von Kandidatenbewerbungen.

In der Wirkungs- oder Resonanzdimension bestanden enge, beidseitige Beziehungen zwischen Beteiligungsarenen und Öffentlichkeit sowie der jeweils übergeordneten Entscheidungsebene, was im Fall der Bürgerforen die Kommission und im Fall der Kommission Bundestag und Bundesregierung waren. Auf beiden Ebenen wurde der Resonanzstandard der Partizipation umfassend erfüllt. Im Zuge der Analyse wurden die zentralen Rückkopplungsmechanismen herausgearbeitet, durch die die Arenen jeweils eingebettet wurden und ihre Vorschläge potenziell Einfluss entwickeln konnten. Hervorzuheben sind:

1. Die Entsendung von „Botschaftern“ aus der Kommission in die Bürgerforen und Online-Diskussionen, wo diese die Kommissionsarbeit erklärten und Rechenschaft ablegten, aber auch wiederum die Vermittlung der Sicht der Bürger gegenüber der Kommission durch diese Botschafter.

2. Die Einbindung von Regierungsvertretern als Beobachter in der Kommission sowie von Mitgliedern des Bundestages als Kommissionsmitgliedern, die zwischen Kommission und Regierungssystem vermittelten.

3. Die Erstellung von übersichtlichen und detailreichen Tabellendarstellungen, die sowohl den Input aus den Bürgerforen als auch den Umgang der Kommission damit für die Öffentlichkeit nachvollziehbar machen.

4. Die uneingeschränkte Transparenz der Kommissionsverhandlungen durch die mit der Besuchsoption verknüpfte unmittelbare Beobachtung der Kommissionsitzungen sowie die Veröffentlichung von Videoaufnahmen und Wortprotokollen aller Sitzungen.

Durch diese Rückkopplungskanäle und Feedbackschleifen hat der Beteiligungsprozess an der Endlagersuche erfolgreich Resonanz erzeugen und Transparenz und Rechenschaft herstellen können.

Auch in Bezug auf die Durchsetzbarkeit der Vorschläge schneiden beide Ebenen gut ab, es lohnt sich aber, hinsichtlich der einzelnen Indikatoren zu unterscheiden: Während die Kommissionvorschläge äußerst konkret und policy-relevant waren und entsprechend sehr weitgehend in die Gesetzgebung übernommen wurden, gab es Abstriche in Bezug auf den erreichten Konsensgrad, denn schließlich scherte der Umweltverband BUND aus. Gemessen an dem Ausmaß des Konflikts und der Tiefe der Gräben zwischen den Lagern von Gorlebenbefürwortern und -gegnern kann dennoch das erreichte Ergebnis als erstaunlich konsensual bezeichnet werden, denn es wurde eine breite Basis erreicht, auf der der Prozess voranschreiten kann.

Man sollte sich allerdings nicht täuschen und das Ergebnis mit allumfassender Zustimmung aller gleichsetzen (Krick 2017). Was die Kommission selbst als ,,breiten Konsens“ und „Einvernehmen“ bezeichnet, ist durch zahlreiche Konfliktminimierungsmechanismen ermöglicht worden (s. auch Krick 2013). Die Analyse zeigt, aufgrund welcher institutionellen Mechanismen und Regeln das Ergebnis zustande kommen konnte, obwohl der Prozess konflikthaft war und auch antagonistische Interessen einbezog, die Kommission breit zusammengesetzt war, über besonders 
konflikthafte Fragen mehrheitlich abgestimmt wurde und selbst am Ende keineswegs zu allen Punkten Einigkeit bestand:

1. Es wurde eine Aggregationsregel angewandt, die man als Konsens-minus-eins bezeichnen könnte ${ }^{18}$ und die auf einem Konsensverständnis aufbaut, das nicht mit Einstimmigkeit gleichzusetzen ist, sondern eher weitgehende Einigkeit bedeutet.

2. Die Gruppe wurde durch die kollektive Verpflichtung auf das Konsensziel, das in der Geschäftsordnung kodifiziert war, zur Kooperationsbereitschaft diszipliniert.

3. Abstimmungen erfolgten nur zu besonders konflikthaften Punkten und beruhten auf einem Zweidrittel-Quorum, und damit auf übergroßen Mehrheiten (die einigen Verständnissen von Konsens durchaus entsprechen).

4. Die Beteiligungsrechte waren dahingehend abgestuft, dass über das Endergebnis nur die Nicht-Politiker unter den Kommissionsmitgliedern abstimmen durften, während die gesamte Kommission aber den Entwurf dieses Ergebnisses vorbereitet und im Einzelnen abgestimmt hatte.

5. Es wurden Sondervoten in den Bericht integriert, die es einzelnen Mitgliedern möglich machten, ihren Unmut über einzelne Aspekte öffentlich zu machen, ohne sich der konsensualen Nostrifizierung des Gesamtberichts entziehen zu müssen.

Im Gegensatz zum Kommissionsergebnis handelte es sich bei den Bürgervorschlägen um eine Vielzahl an Stimmen aus unterschiedlichen Foren, die nicht zu einem gemeinsam abgestimmten, konsensualen Bericht und damit einer klaren Botschaft an die übergeordnete Ebene zusammengeführt wurden. Die Aggregation der Stimmen wurde dennoch geleistet durch die Erstellung sehr detaillierter Ergebnisprotokolle und verdichteter Übersichtstabellen aller Vorschläge. Das war in diesem Fall ein zweckdienlicher Aggregationsmechanismus, der eine hohe Transparenz zuließ. Dennoch hätte der Einfluss der Bürgerinputs vermutlich erhöht werden können, wenn Abstimmungsprozesse über die Grenzen einzelner Bürgerforen hinaus existiert hätten.

Insgesamt unterstreicht die vorliegende Studie, wie anspruchsvoll eine sinnvolle, normativ befriedigende, demokratisierende Ausweitung politischer Beteiligung ist und dass dies bei höchstem Einsatz zwar möglich, aber auch außerordentlich ressourcenaufwändig ist. Wenngleich dies also im großen Stil für alle (oder auch nur die umstrittensten) politischen Fragen nicht reproduzierbar sein mag, können aus der Fallstudie doch Lehren gezogen werden zur Realisierung inklusiver und wirksamer Beteiligung - und vor allem zum Umgang mit den häufig betonten Schwächen gerade innovativer Beteiligungsformen: dem sozialen Bias und der mangelnden Durchschlagskraft.

Die Studie verweist auch darauf, dass selbst ein hohes Maß an Inklusivität und Wirksamkeit keineswegs die Zufriedenheit der Beteiligten und der Öffentlichkeit mit dem Partizipationsprozess garantiert - sei es, weil unerreichbare und inkonsistente Vorstellungen von Beteiligung zum Maßstab erhoben werden, weil das „Erwar-

18 Die sogenannte Konsens-minus-eins-Regel findet in einigen informellen Konsensprozessen Anwendung und ist teilweise auch in internationalen Organisationen kodifiziert, beispielsweise im Rahmen der OSZE. 
tungsmanagement" mangelhaft war oder weil die zugrunde liegenden Probleme (die Endlagerung atomaren Mülls) keine befriedigende Lösung erlauben.

Funding Open Access funding provided by University of Oslo (incl Oslo University Hospital).

Open Access Dieser Artikel wird unter der Creative Commons Namensnennung 4.0 International Lizenz veröffentlicht, welche die Nutzung, Vervielfältigung, Bearbeitung, Verbreitung und Wiedergabe in jeglichem Medium und Format erlaubt, sofern Sie den/die ursprünglichen Autor(en) und die Quelle ordnungsgemäß nennen, einen Link zur Creative Commons Lizenz beifügen und angeben, ob Änderungen vorgenommen wurden.

Die in diesem Artikel enthaltenen Bilder und sonstiges Drittmaterial unterliegen ebenfalls der genannten Creative Commons Lizenz, sofern sich aus der Abbildungslegende nichts anderes ergibt. Sofern das betreffende Material nicht unter der genannten Creative Commons Lizenz steht und die betreffende Handlung nicht nach gesetzlichen Vorschriften erlaubt ist, ist für die oben aufgeführten Weiterverwendungen des Materials die Einwilligung des jeweiligen Rechteinhabers einzuholen.

Weitere Details zur Lizenz entnehmen Sie bitte der Lizenzinformation auf http://creativecommons.org/ licenses/by/4.0/deed.de.

\section{Literatur}

Arnstein, Sherry R. 1969. A ladder of citizen participation. Journal of the American Planning Association 35(4):216-224.

Berlin Institut für Partizipation. 2018. Das Konzept der Partizipationsleiter. https://www.bipar.de/daskonzept-der-partizipationsleiter/. Zugegriffen: 20.11.2020.

Brady, Henry, Sydney Verba, und Kay L. Schlozman. 1995. Beyond SES: a resource model of political participation. American Political Science Review 89(2):271-294.

Brown, Mark B. 2006. Survey article. Citizen panels and the concept of representation. The Journal of Political Philosophy 14(2):203-225.

Brunnengräber, Achim. 2016. Die atompolitische Wende. Paradigmenwechsel, alte und neue Narrative undKräfteverschiebungen im Umgang mit radioaktiven Abfällen. In Problemfalle Endlager, Gesellschaftliche Herausforderungen im Umgang mit Atommüll, Hrsg. Achim Brunnengräber, 13-32. Baden-Baden: Nomos.

BUND. 2015. 1 Jahr Atommüll-Kommission. Die Zwischenbilanz des Bund für Umwelt und Naturschutz Deutschland (BUND), Stellungnahme vom 21.05.2015

Bundesamt für kerntechnische Entsorgungssicherheit (BfE; heute BASE). 2019. Information, Dialog, Mitgestaltung. Öffentlichkeitsbeteiligung in der Startphase der Endlagersuche, Konzeptentwurf, Stand: April 2019. https://www.base.bund.de/SharedDocs/IP6/BASE/DE/20190403_OEB_Konzept_ ueberarbeitet.pdf?_blob=publicationFile\&v=9. Zugegriffen: 20.11.2020

Bürgerinitiative Umweltschutz Lüchow Dannenberg, und Arbeitsgemeinschaft Schacht Konrad/ ausgestrahlt.de.. 2016. Atommüll-Kommission am Ende. Konflikte ungelöst. Ein Reader zum Abschlussbericht der ,Kommission Lagerung hoch radioaktiver Abfallstoffe

Demos/Prognos. 2015. Beteiligungskonzept. Konzept für die Beteiligung der Öffentlichkeit am Bericht der Kommission Lagerung hoch radioaktiver Abfallstoffe. Berlin (Kommissions-Drucksache (K-Drs.) 108 neu). https://www.bundestag.de/endlager-archiv/blob/383266/90c691f0815a09f8d160c8f4fc973 8bb/drs_108-neu-data.pdf.. Zugegriffen: 20.11.2020

van Deth, Jan W. 2009. Politische Partizipation. In Politische Soziologie. Ein Studienbuch, Hrsg. Viktoria Kaina, Andrea Römmele, 141-161. Wiesbaden: VS.

Deutsche Arbeitsgemeinschaft Endlagerforschung. 2016. Partizipation im Standortauswahlverfahren für ein Endlager. http://www.daef2014.org/DAEF/assets/daef-partizipation_2016-03_web-1-.pdf. Zugegriffen: 20.11.2020.

Dialogik/EIPP. 2016. Evaluationsbericht zum Beteiligungsverfahren der Kommission Lagerung hoch radioaktiver Abfallstoffe., im Auftrag der Kommission Lagerung hoch radioaktiver Stoffe. 22.02.2016 (K.-Drs. 230). https://www.bundestag.de/endlager-archiv/blob/423458/6003ee0ca05983f27d89d6b0 e6e267c9/drs_230-data.pdf. Zugegriffen: 20.11.2020. 
Dryzek, John S. 2001. Legitimacy and economy in deliberative democracy. Political Theory 29(5):651-669. Endlagerdialog.de. 2018. Öffentlichkeitsbeteiligung nach StandAG. https://endlagerdialog.de/2018/12/ ausgestrahlt-oeffentlichkeitsbeteiligung-standag/\#more-10202. Zugegriffen: 20.11.2020.

Endlagerkommission. 2016. Abschlussbericht der Kommission Lagerung hoch radioaktiver Abfallstoffe (K.Drs. 268). https://www.bundestag.de/endlager-archiv/blob/434430/bb37b21b8e1e7e049ace5db6b 2f949b2/drs_268-data.pdf. Zugegriffen: 20.11.2020.

Fung, Archon. 2003. Survey article: recipes for public spheres: eight institutional design choices and their consequences. The Journal of Political Philosophy 11(3):338-367.

Fung, Archon. 2006. Varieties of participation in complex governance. Public Administration Review 66(1):66-74.

Fung, Archon. 2013. The principle of affected interests and inclusion in democratic governance. In Representation: elections and beyond, Hrsg. Jack H. Nagel, \& Rogers M. Smith, 236-268. Philadelphia: University of Pennsylvania Press.

Gabriel, Oskar, und Kerstin Völkl. 2005. Politische und Soziale Partizipation. In Handbuch Politisches System der Bundesrepublik Deutschland, 3. Aufl., Hrsg. Oscar W. Gabriel, Everhard Holtmann, 523-573. München, Wien: Oldenbourg.

Haas, Peter M. 2004. When does power listen to truth? A constructivist approach to the policy process. Journal of European Public Policy 11(4):569-592.

Irvin, Renée A., und John Stansbury. 2004. Citizen participation in decision making: Is it worth the effort? Public Administration Review 64(1):55-65.

Kalmbach, Karena. 2016. Ein Forum zur Entwicklung neuer Lösungsansätze oder zur Austragung alter Konflikte? In Problemfalle Endlager, Gesellschaftliche Herausforderungen im Umgang mit Atommüll, Hrsg. Achim Brunnengräber, 389-408. Baden-Baden: Nomos.

Kamlage, Jan-Hendrik, Jan Warode, und Anna Mengede. 2019. Chances, challenges and choices of participation in siting a nuclear waste repository. The German case. In Conflicts, participation and acceptability in nuclear waste governance An International Comparison Volume, Bd. 3, Hrsg. Achim Brunnengräber, Rosaria Maria Di Nucci, 91-110. Wiesbaden: VS.

Kostka, Dieter. 2018. Öffentlichkeitsbeteiligung bei der Standortsuche für ein langfristiges Atommüll-Lager nach StandAG. https://www.ausgestrahlt.de/media/Studie_Dieter_Kostka_\%C3\%96ffentlichkeits beteiligung.pdf. Zugegriffen: 20.11.2020.

Krick, Eva. 2013. Verhandlungen im Konsensverfahren. Wiesbaden: VS.

Krick, Eva. 2014. Partizipationspotentiale von Expertengremien. Der Trade-off zwischen Inklusion und Effektivität im Verhandlungsprozess. Österreichische Zeitschrift für Politikwissenschaft 43(1):7-22.

Krick, Eva. 2017. The myth of effective veto power under the rule of consensus. Dynamics and democratic legitimacy of collective decision-making by 'tacit consent. Revue Négociations 27(1):109-128.

Krick, Eva. 2018. The epistemic quality of expertise. Contextualised criteria for the multi-source, negotiated policy advice of stakeholder fora. Critical Policy Studies 12(2):209-226.

Krick, Eva. 2019. Creating participatory expert bodies. How the targeted selection of policy advisers can bridge the epistemic-democratic divide. European Politics and Society 20(1):101-116.

Krick, Eva, und Cathrine Holst. 2018. Committee governance in consensus cultures: an exploration of best practice cases in Germany and Norway. In Democratic state and democratic society: institutional change in the Nordic model, Hrsg. Fredrik Engelstad, Cathrine Holst, und Gunnar C. Aakvaag, 151-174. Warschau: De Gruyter.

Kuppler, Sophie. 2016. Modellfalle Schweiz. Was aus der Standortsuche gelernt und generalisiert werden kann. In Problemfalle Endlager, Gesellschaftliche Herausforderungen im Umgang mit Atommüll, Hrsg. Achim Brunnengräber, 339-358. Baden-Baden: Nomos.

Lafont, Christina. 2015. Deliberation, participation and democratic legitimacy: should deliberative minipublics shape public policy? The Journal of Political Philosophy 23(1):40-63.

Lentsch, Justus, und Peter Weingart. 2011. Quality control in the advisory process: towards an institutional design for robust science advice. In The politics of scientific advice: institutional design for quality assurance, Hrsg. Justus Lentsch, Peter Weingart, 353-374. Cambridge: Cambridge University Press.

Lijphart, Arend. 1997. Unequal participation. Democracy's unresolved dilemma. American Political Science Review 91(1):1-14.

Losada, Ana María Isidoro, Dörte Themann, und Maria Rosaria Di Nucci. 2019. Experts and politics in the German nuclear waste governance. Advisory bodies between ambition and reality. In Conflicts, Participation and Acceptability in Nuclear Waste Governance. An International Comparison, Bd. 3, Hrsg. Achim Brunnengräber, Rosaria Maria Di Nucci, 231-259. Wiesbaden: Verlag für Sozialwissenschaften. 
Mansbridge, Jane, et al. 2012. A systematic approach to deliberative democracy. In Deliberative systems: deliberative democracy at the large scale, Hrsg. John Parkinson, Jane Mansbridge, 1-26. Cambridge: Cambridge University Press.

Marien, Sofie, Marc Hooghe, und Ellen Quintelier. 2010. Unconventional participation and the problem of inequality: a comparative analysis. In New forms of citizen participation. Normative implications, Hrsg. Erik Amnå, 131-146. Baden-Baden: Nomos.

Di Nucci, Maria Rosaria. 2016. NIMBY oder IMBY Akzeptanz, Freiwilligkeit und Kompensationen in der Standortsuche für die Endlagerung radioaktiver Abfälle. In Problemfalle Endlager, Gesellschaftliche Herausforderungen im Umgang mit Atommüll, Hrsg. Achim Brunnengräber, 119-144. Baden-Baden: Nomos.

Pateman, Carole. 1970. Participation and democratic theory. Cambridge: Cambridge University Press.

Pitkin, Hanna F. 1967. The concept of representation. Berkeley, Los Angeles: University of California Press.

Ritzi, Claudia, und Gary S. Schaal. 2014. Legitimitätsgenerierung im deliberativen und repräsentativen Paradigma: Komplementär oder kompetitiv? In Deliberative Demokratie in der Diskussion. Herausforderungen, Bewährungsproben, Hrsg. Claudia Landwehr, Rainer Schmalz-Bruns, 239-272. BadenBaden: Nomos.

Ritzi, Claudia, und Wagner, Aiko. 2016. Symbolisch oder echt? Die politische Beteiligung junger Erwachsener in Deutschland online und offline. Zeitschrift für Parlamentsfragen, 47(2), 304-326.

Rowe, Gene, und Lynn J. Frewer. 2000. Public participation methods: a framework for evaluation. Science, Technology and Human Values 25(3):3-29.

Ryfe, David M. 2005. Does deliberative democracy work? Annual Review of Political Science 8(1):49-71.

Saward, Michael. 2010. The representative claim. Oxford: Oxford University Press.

Schäfer, Armin, und Harald Schoen. 2013. Mehr Demokratie aber nur für weniger? Der Zielkonflikt zwischen mehr Beteiligung und politischer Gleichheit. Leviathan 41(1):94-120.

Seier, Sebastian. 2016. Mal mehr, mal weniger Partizipation. Die Suche nach einem Atommüll-Endlager in Frankreich und Schweden im Vergleich. In Problemfalle Endlager, Gesellschaftliche Herausforderungen im Umgang mit Atommüll, Hrsg. Achim Brunnengräber, 359-387. Baden-Baden: Nomos.

Setälä, Maija. 2017. Connecting deliberative mini-publics to representative decision making. European Journal of Political Research 56:846-863.

Stolle, Martin. 2006. Die Einstellung zur Endlagerung und die politische Partizipation der Bevölkerung. Ergebnisse zweier repräsentativer Bevölkerungsumfragen. In Wohin mit dem radioaktiven Abfall? Perspektiven für eine sozialwissenschaftliche Endlagerforschung, Hrsg. Peter Hocke-Bergler, Armin Grunwald, 193-216. Berlin: Edition sigma.

Urbinati, Nadia, und Marc E. Warren. 2008. The concept of representation in contemporary democratic theory. Annual Review of Political Sciences 11(1):387-412.

Warren, Mark E. 2002. What can democratic participation mean today? Political Theory 30(5):677-701.

Young, Iris M. 2000. Inclusion and democracy. Oxford: Oxford University Press. 